\title{
Polyamine Transport Inhibitor AMXT-1501 Dicaprate
}

National Cancer Institute

\section{Source}

National Cancer Institute. Polyamine Transport Inhibitor AMXT-1501 Dicaprate. NCI

Thesaurus. Code C148487.

The dicaprate salt form of AMXT-1501, an orally bioavailable polyamine transport inhibitor, with immunostimulating and antineoplastic activities. Upon administration, AMXT-1501 targets, binds to and blocks polyamine transport from the bloodstream into the tumor microenvironment (TME), thereby preventing cancer cell uptake. This decreases polyamine concentrations inside the TME and tumor cell, inhibits tumor cell proliferation and induces apoptosis. In addition, AMXT-1501 may abrog ate polyaminemediated immune suppression in the TME. Polyamines, naturally found in normal, heathy cells, are required for normal cell growth and division. In cancer cells, polyamines play key roles in tumor cell proliferation and tumor-induced suppression of the patient's immune system. 